\title{
BRAMS: the Belgian RAdio Meteor Stations
}

\author{
H. Lamy • S. Ranvier • J. De Keyser • S. Calders • E. Gamby • C. Verbeeck
}

\begin{abstract}
In the last months, the Belgian Institute for Space Aeronomy has been developing a Belgian network for observing radio meteors using forward scattering technique. This network is called BRAMS for Belgian RAdio Meteor Stations. Two beacons emitting a circularly polarized pure sine wave toward the zenith act as the transmitters at frequencies of 49.97 and $49.99 \mathrm{MHz}$. The first one located in Dourbes (Southern Belgium) emits a constant power of 150 Watts while the one located in Ieper (Western Belgium) emits a constant power of 50 Watts. The receiving network consists of about 20 stations hosted mainly by radio amateurs. Two stations have crossed-Yagi antennas measuring horizontal and vertical polarizations of the waves reflected off meteor trails. This will enable a detailed analysis of the meteor power profiles from which physical parameters of the meteoroids can be obtained. An interferometer consisting of 5 Yagi-antennas will be installed at the site of Humain in order to determine the angular detection of one reflection point, allowing us to determine meteoroid trajectories. We describe this new meteor observing facility and present the goals we expect to achieve with the network.
\end{abstract}

Keywords radio meteors $\cdot$ forward scattering

\section{Introduction}

The Earth's atmosphere is constantly hit by thousands of meteoroids with sizes ranging from submillimeters to several meters. Their estimated cumulative mass is in the range [40-100] tons per day. They play a crucial role in a number of astronomical and aeronomical studies and, given their intercept velocities in excess of $11 \mathrm{~km} / \mathrm{s}$, they pose a significant threat to spacecraft. Traditionally, they have been detected by visual means or with radars during their interaction with the atmosphere. Here we propose to study the meteoroid population with the BRAMS (Belgian RAdio Meteor Stations) network, a set of radio receiving stations using forward scattering techniques and two dedicated beacons as transmitters. The BRAMS network will be finished by the end of 2010. Its current state is described in section 2 while section 3 is devoted to the objectives of the project. In the conclusion, the advantages of a forward scattering system over traditional radar systems will be shortly discussed.

\section{The BRAMS Network}

In 2009 the Belgian Institute for Space Aeronomy (BISA) initiated the development of BRAMS, a Belgian network of radio receiving stations using forward scattering techniques to detect meteors. This

H. Lamy $(\square) \cdot$ S. Ranvier • J. De Keyser • S. Calders $\bullet$ E. Gamby

Belgian Institute for Space Aeronomy, Avenue Circulaire 3, 1180 Brussels, Belgium. E-mail: herve.lamy@aeronomie.be

C. Verbeeck

Royal Observatory of Belgium, Avenue Circulaire 3, 1180 Brussels, Belgium 
project is carried out in collaboration with about 20 Belgian radio amateurs or groups of amateur astronomers which will host several stations throughout the country (see black squares on Figure 1).

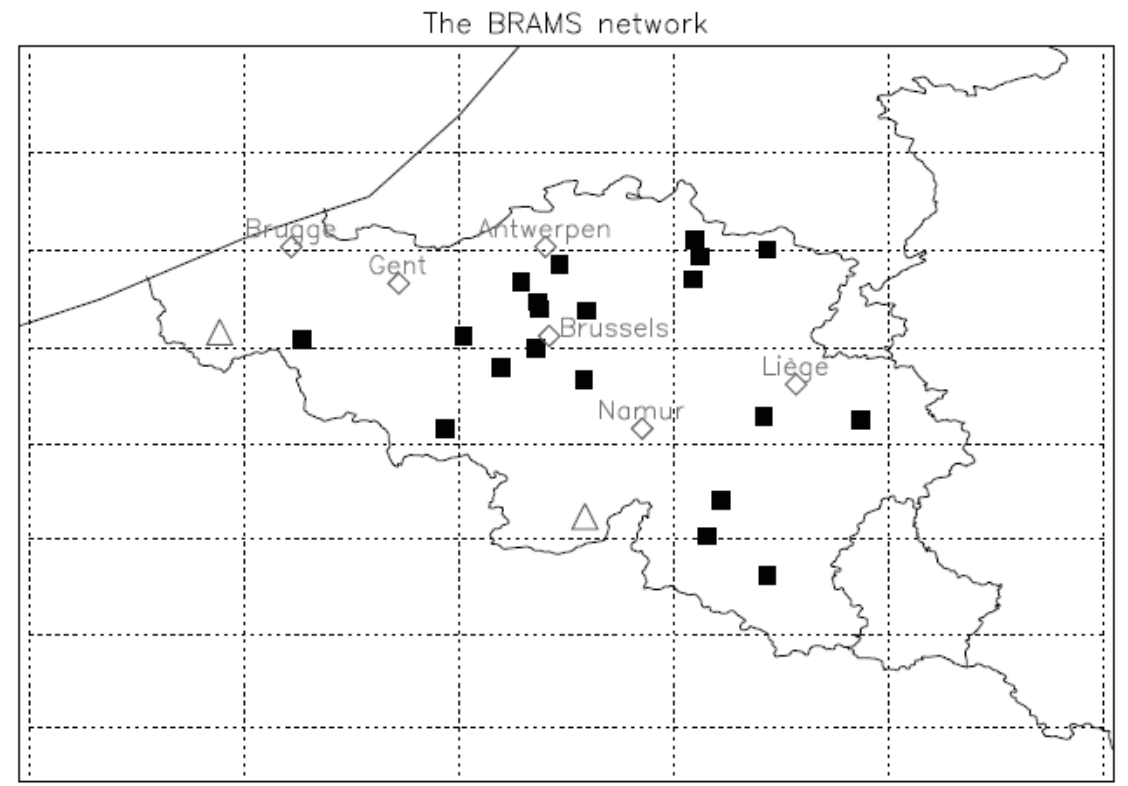

Figure 1. Geographical distribution of the stations of the BRAMS network. The black squares represent the receiving stations while the two triangles represent the two beacons (Ieper in the West and Dourbes in the SouthEast). The biggest cities of Belgium, represented by rhombs, have been added to facilitate identification of the stations.

The transmitters are two dedicated beacons located in Ieper and Dourbes (the two triangles on Figure 1). They emit a pure sinusoidal wave with a constant power of respectively 50 and 150 watts, at frequencies respectively of 49.99 and $49.97 \mathrm{MHz}$. These frequencies are protected avoiding ambiguity about the origin of detected meteor echoes. The beacon in Ieper is a crossed 2-element Yagi antenna which has been constructed in 2008 by radio amateurs from the VVS (Vereniging Voor Sterrenkunde Flemish association of amateur astronomers). It emits toward the zenith with a HPBW (Half-Power Beam Width) of $\sim 100^{\circ}$ (see Figure 2) which covers a large surface of the sky at altitudes between 90 and $120 \mathrm{~km}$. The theoretical total gain of this antenna is $5.9 \mathrm{dBi}$. The beacon in Dourbes is under construction and will be active in September 2010. It will be similar to the one in Ieper but will use a $8 \mathrm{~m} \times 8 \mathrm{~m}$ grid as the reflector to increase the power emitted upward (HPBW $\sim 64^{\circ}$-see Figure 2 ). With this grid, the total gain is increased by $3.6 \mathrm{~dB}$ as compared to the Ieper beacon. The stability of the frequency of the Dourbes beacon is secured with a GPS disciplinated OCXO (Oven-Controlled Crystal Oscillator) $10 \mathrm{MHz}$ reference.

A typical receiving station is made of a 3-element Yagi antenna linked to a ICOM ICR75 receiver by a coaxial cable. The received signal is sampled and stored on a local PC. With 20 receiving stations spread over Belgium, we will increase both the number of meteor detections and the chances of having the same meteor detected simultaneously by several stations. This last point is essential for the determination of meteoroid trajectories since each detection gives information only about one point of the trajectory. This requires a very good synchronization of the stations. The stations will be synchronized by using the NTP (Network Time Protocol) application and a local GPS as reference clock. Accuracy of $1 \mathrm{~ms}$ is expected. Each station will generate approximately $1 \mathrm{~GB}$ of data per day. First these data will be temporarily stored on local hard disks then later archived with the IT facilities of 


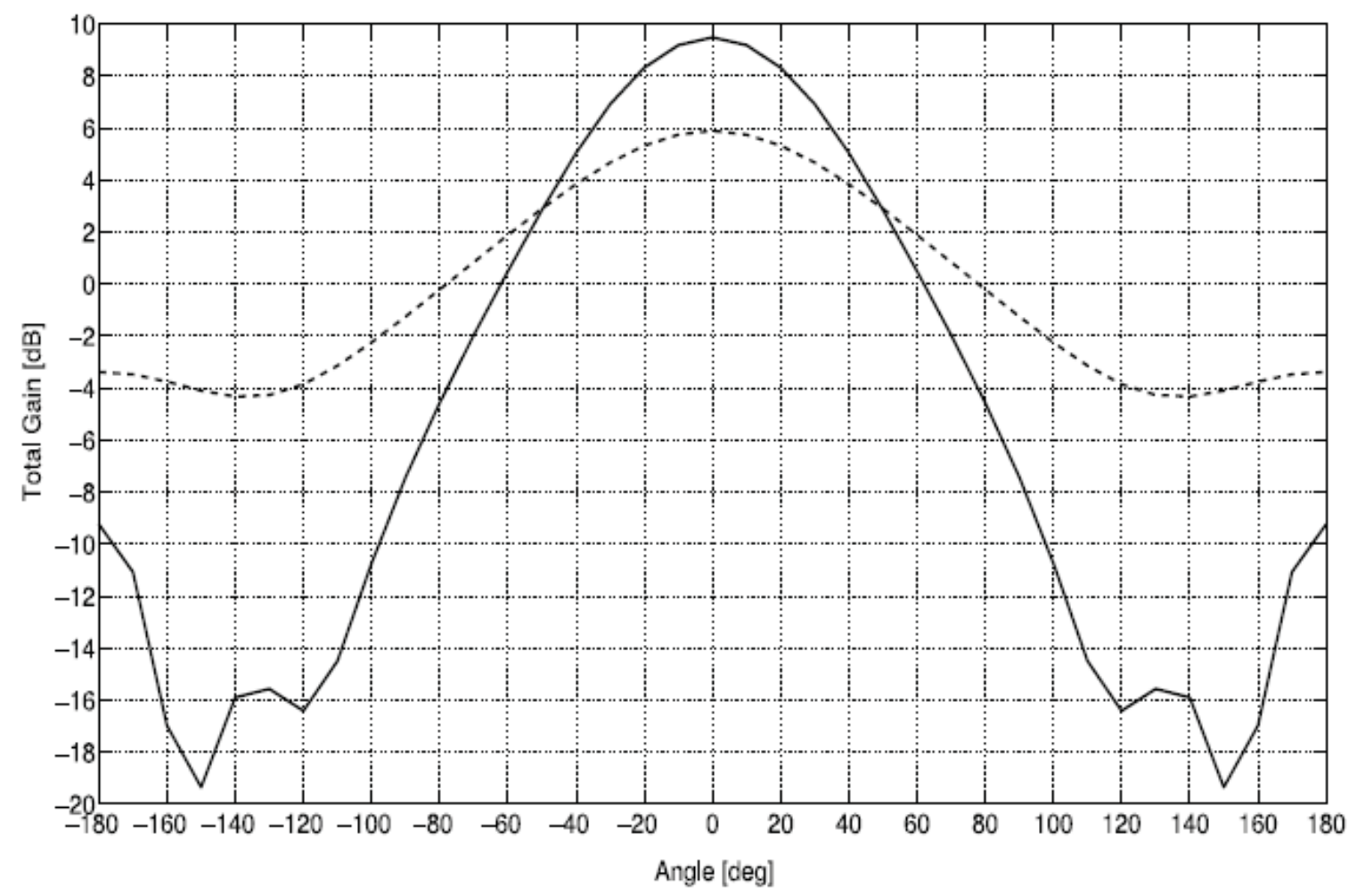

Figure 2. Theoretical radiation pattern (in $\mathrm{dB}$ ) of the two beacon antennas (circularly polarized). Solid line: Dourbes beacon (with a $8 \mathrm{~m} \times 8 \mathrm{~m}$ grid). Dashed line: Ieper beacon.

BISA, where all data will be analyzed and become available to the scientific community. In these data there will be a large number of meteor echoes but also a large number of "spurious" echoes such as reflection of radio waves on planes, sporadic Es, thunderstorms, etc. as well as local broad-band interferences. A software is currently developed to automatically select meteor echoes only.

The station in BISA (located in Uccle in the South of Brussels) is also equipped with an additional antenna, a crossed 3-element Yagi, connected to a low noise amplifier (MITEQ AU-1138) and a software defined radio receiver (USRP2). With this setup, we will measure both polarizations (horizontal and vertical) of the meteor echoes and combine them to obtain the total power reflected by the meteor. This will allow us to perform quantitative analyses of the signal in order to retrieve physical parameters of the meteoroids.

An interferometer made of 5 Yagi antennas will be installed in the radio astronomical site of Humain in order to determine the angular direction of one reflection point with an accuracy $\leq 1-2^{\circ}$. Humain is located at approximately $60 \mathrm{~km}$ north-east of Dourbes. We will use the 5 antenna configuration of [8] consisting of two orthogonal linear arrays of 3 antennas with the central one common to both arrays. The central antenna will be a crossed 3-element Yagi antenna used in order to obtain the total power of the incoming waves like in the BISA station. To get information on the angular direction of the reflection point, the phase of the signals from the 5 antennas are compared. For this purpose, the analog-to digital converters of the five USRP2 receivers are synchronized. In addition, the frequency stability of the receivers is ensured by a $10 \mathrm{MHz}$ reference similar to the one used for the beacon in Dourbes. 


\section{Objectives of the Project}

By using the BRAMS network, we plan to reach the objectives described below. They are separated according to whether they can be achieved with a single station, with several stations or with several stations and the interferometer.

1. By using data from a single receiving station, the activity profiles of meteor showers (number of echoes versus time) can be obtained by subtracting sporadic meteor echoes from all echo counts. These activity profiles must be corrected for the observability function of the station, which describes its sensitivity for detecting meteoroid echoes from a specific direction in the sky ([7], [15], [13]). The activity profiles of the main meteor showers will be studied and compared to results in the literature. Mass indexes and flux densities will be calculated for meteor showers and sporadic background using the method developed by [1] and [11], [12].

2. Simultaneous detection of a meteor by several stations allows in principle to retrieve some information about the meteoroid trajectory. We will test a method proposed by [9] exploiting the fact that a meteor trail is tangential to a family of ellipsoids with the receivers as one focal point and the transmitter as the other one (specularity condition). If the meteor is also detected by the interferometric station in Humain, the problem is simplified since then we know the angular direction of the reflection point for this particular geometry. The height of this reflection point can be obtained for shower meteors but is still unknown for sporadic meteors. However, for underdense meteors, it can be estimated from the exponential decay of the power profile (with the use of a good atmospheric model such as MSISE-00) and for overdense meteors, it can be estimated with the method proposed by [5]. With the 3-D position of one specular point and with detections of the meteor by other stations, we can retrieve the trajectory of the meteoroid even for sporadic meteors ([16]). Eventually the goal is to produce a map in Sun-centered ecliptical coordinates of the distribution of meteor radiants and to compare it to similar ones obtained by optical or radar means (e.g. [4]).

3. For a given meteor, when the geometry is fully resolved, the study of the power profiles give access to several important physical parameters if the technical characteristics of the receiving station (antenna gains, polarization of the reflected wave, calibration of the acquisition card, etc.) are perfectly known [16]. These characteristics will only be known for the stations in BISA and in Humain. For the others, we will only have reasonable estimates. The electron line density at the reflection point can be computed from the maximum of the power profile. If the electron line density is obtained in several points of the meteoroid trail (from multi-station observations), the initial mass of the meteoroid can be estimated with models of meteoroid ablation in the Earth's atmosphere (e.g. [3]). The ambipolar diffusion coefficient can be obtained from the exponential decay of underdense meteors and yields information on the meteor's height. The speed of the meteoroid can be determined by several methods: a) from Fresnel oscillations if these are present and if the signal-to-noise ratio of the data is large enough, $b$ ) from initial rise times of the power profiles if the meteor is observed by several stations (requiring a good synchronization from the various stations) and c) from the Doppler effect for head echoes (see below).

4. Head echoes are associated with the ionized region in front of the meteoroid. Therefore, these echoes in spectrograms show a large Doppler effect due to the high velocity component of the 
object along the line-of-sight. If such an echo is detected by at least 3 stations, we can in principle retrieve the total velocity of the meteoroid [10], [14]. In combination with an ablation model, the meteoroid mass and density can be estimated. Comparison with the results on head echoes obtained with High-Power Large-Aperture radars will be considered.

5. Once we have the radiant of individual meteors and also an estimate of the relative intercept velocity of the incoming particle, the next step is to calculate the orbital parameters of the detected meteoroids. A correction must be applied for the acceleration produced by the gravitational focusing of Earth as well as for the Earth's heliocentric velocity. Knowing the orbital parameters contributes to a better understanding of the distribution and evolution of material in the Solar System.

6. Another advantage of having stations with dual polarized antennas is the ability to investigate the polarization of the received waves. Since most of receiving stations can detect only one polarization, it is very important to determine the depolarization coefficient of the reflections on the meteor trail. The rotation of the polarization plane of the incoming wave can be due to ionospheric Faraday rotation or as a result of the scattering from meteor trails. We will investigate whether the depolarization depends on the type, the trajectory, or the size of the meteor trail. To our knowledge, very few similar measurements have been done with a forwardscatter system (see however [2]).

\section{Conclusion}

The BRAMS network will be a very useful tool to better characterize the distribution of meteoroids in the Solar System. Most researchers employ a backscatter set-up rather than a forward-scatter system, as the latter has a much more complicated geometry. However, backscatter systems suffer from the echo ceiling selection effect, which limits their views on faint and fast meteors. Forward-scatter setups are much less vulnerable to this selection effect, hence yielding a less biased meteoroid population. To our knowledge, there are only two other forward-scatter systems run by professional astronomers: the Bologna-Lecce-Modra system [6] and the HRO system at the Kochi University in Japan [17]. The former has only 2 receiving stations and no interferometric capabilities while the latter has only 6 stations. BRAMS will feature at least 20 stations that will allow improved multi-station analysis and will also have the additional advantage of an interferometric system.

\section{Acknowledgements}

BRAMS is a project of the Belgian Institute for Space Aeronomy which is funded by the Belgian SolarTerrestrial Center of Excellence (STCE -http://www.stce.be). This project is carried out in collaboration with many radio amateurs from Belgium, in particular with several members from the VVS (Vereniging Voor Sterrenkunde). We would like to thank them for their participation in this project. 


\section{References}

1. Belkovich O. et al., Processing of radar observations, Proceedings of the Radio Meteor School 2005, Oostmalle, Eds. C. Verbeeck and J.-M. Wislez, (2006).

2. Billam E.R. \& Browne I.C., Characteristics of Radio Echoes from Meteor Trails IV: Polarization Effects, Proceedings of the Physical Society, section B, Volume 69, Number 1, (1955).

3. Campbell-Brown M.D., Koschny D., Model of the ablation of faint meteors, A\&A, 418, 751-758, (2004).

4. Campbell-Brown M.D., High Resolution radiant distribution and orbits of sporadic radar meteoroids, Icarus 196, 144163, (2008).

5. Carbognani A., De Meyere M., Foschini L., Steyaert C., On the meteor height from forward scatter radio observations, A\&A,361, 293-297, (2000).

6. Cevolani G. et al, Baseline effect on the forward scatter radar reflections from meteor trains, Il Nuovo Cimento C, 19, 447-450, (1996)

7. Hines C.O., Diurnal variations in the number of shower meteors detected by the forward-scattering of radio waves -Part III. Ellipsoidal theory, Can. J. Phys., 36, 117-126 (1958).

8. Jones J., Webster A.R., Hocking W.K., An improved interferometer design for use with meteor radars, Radio Sci., 33, 55-65 (1998).

9. Nedeljkovic S., Meteor forward scattering at multiple frequencies, Proceedings of the Radio Meteor School 2005, Oostmalle, Eds. C. Verbeeck and J.-M. Wislez, (2006).

10. Richardson J. \& Kuneth W., Revisiting the radio Doppler effect from forward-scatter meteor head echoes, WGN, Journal of the International Meteor Organization, 26, 117-130, (1998).

11. Ryabova G.O., Calculation of the incident flux density of meteors by numerical integration I, WGN, Journal of the International Meteor Organization, 36, 120-123, (2008).

12. Ryabova G.O., Calculation of the incident flux density of meteors by numerical integration II, WGN, Journal of the International Meteor Organization, 37, 63-67, (2009).

13. Steyaert C., Brower J., Verbelen F., A numerical method to aid in the combined determination of stream activity and Observability Function, WGN, Journal of the International Meteor Organization, 34, 87-93, (2006).

14. Steyaert C., Verbelen F. et al, Meteor Trajectory from Multiple Station Head Echo Doppler Observations, submitted to WGN.

15. Verbeeck C., Calculating the sensitivity of a forward scatter setup for underdense shower meteors, Proceedings of the International Meteor Conference 1996 (Eds. A. Knöfel and P. Roggemans), 122-132 (1997).

16. Wislez J-M., Meteor astronomy using a forward scatter set-up, Proceedings of the Radio Meteor School 2005, Oostmalle, Eds. C. Verbeeck and J.-M. Wislez, (2006).

17. Yamamoto M.-Y. et al, Development of HRO interferometer at Kochi University of Technology, Proceedings of the International Meteor Conference 2006, Eds. F. Bettonvil \& J. Kac, 117-125, (2007). 